\title{
Higher Mortality of Ischaemic Stroke Patients Hospitalized with COVID-19 Compared to Historical Controls
}

\author{
Stephanie L. Harrison ${ }^{a}$ b Elnara Fazio-Eynullayeva ${ }^{c}$ Deirdre A. Lane ${ }^{a, b}$ \\ Paula Underhill $^{d}$ Gregory Y.H. Lip ${ }^{a, b}$ \\ aLiverpool Centre for Cardiovascular Science, University of Liverpool \& Liverpool Heart and Chest Hospital, \\ Liverpool, UK; ${ }^{\mathrm{b}}$ Cardiovascular and Metabolic Medicine, Institute of Life Course and Medical Sciences, University of \\ Liverpool, Liverpool, UK; 'TriNetX LLC, Cambridge, MA, USA; ${ }^{\mathrm{d}}$ TriNetX LLC, London, UK
}

\section{Keywords}

Stroke $\cdot$ Cerebrovascular disease $\cdot$ Coronavirus · Coronavirus disease 2019. Mortality

\begin{abstract}
Introduction: Increasing evidence suggests patients with coronavirus disease 2019 (COVID-19) may develop thrombosis and thrombosis-related complications. Some previous evidence has suggested COVID-19-associated strokes are more severe with worse outcomes for patients, but further studies are needed to confirm these findings. The aim of this study was to determine the association between COVID-19 and mortality for patients with ischaemic stroke in a large multicentre study. Methods: A retrospective cohort study was conducted using electronic medical records of inpatients from 50 healthcare organizations, predominately from the USA. Patients with ischaemic stroke within 30 days of COVID-19 were identified. COVID-19 was determined from diagnosis codes or a positive test result identified with $\mathrm{CO}$ VID-19-specific laboratory codes between January 20, 2020, and October 1, 2020. Historical controls with ischaemic stroke without COVID-19 were identified in the period January 20,2019, to October 1,2019. 1:1 propensity score matching was used to balance the cohorts with and without CO-
\end{abstract}

VID-19 on characteristics including age, sex, race and comorbidities. Kaplan-Meier survival curves for all-cause 60-day mortality by COVID-19 status were produced. Results: During the study period, there were 954 inpatients with ischaemic stroke and COVID-19. During the same time period in 2019 , there were 48,363 inpatients with ischaemic stroke without COVID-19 (historical controls). Compared to patients with ischaemic stroke without COVID-19, patients with ischaemic stroke and COVID-19 had a lower mean age, had a lower prevalence of white patients, a higher prevalence of black or African American patients and a higher prevalence of hypertension, previous cerebrovascular disease, diabetes mellitus, ischaemic heart disease, atrial fibrillation, chronic kidney disease, chronic obstructive pulmonary disease, liver disease, neoplasms, and mental disorders due to known physiological conditions. After propensity score matching, there were 952 cases and 952 historical controls; cases and historical controls were better balanced on all included characteristics (all $p>0.05$ ). After propensity score matching, Kaplan-Meier survival analysis showed the survival probability was significantly lower in ischaemic stroke patients with COVID-19 (78.3\% vs. $91.0 \%$, log-rank test $p<0.0001)$. The odds of 60-day mortality were significantly higher for patients with ischaemic stroke and COVID-19 compared to the propensity score-matched historical controls (odds ratio: 2.51

$\begin{aligned} & \text { karger@karger.com } \\ & \text { www.karger.com/ced }\end{aligned}$
Karger ${ }^{\prime /}$


[95\% confidence interval 1.88-3.34]). Discussion/Conclusions: Ischaemic stroke patients with COVID-19 had significantly higher 60-day all-cause mortality compared to propensity score-matched historical controls (ischaemic stroke patients without COVID-19).

(C) 2021 S. Karger AG, Basel

\section{Introduction}

Coronavirus disease 2019 (COVID-19) was declared a public health emergency by the World Health Organization on January 30, 2020 [1]. As of mid-October 2020, over 39.7 million cases of COVID-19 and over 1.1 million deaths with COVID-19 had been reported worldwide [2]. Increasing evidence points to a propensity to thrombosis, and thrombosis-related complications, including venous thromboembolism and arterial thrombosis for COVID-19 patients [3]. A series of 6 case reports suggested greater ischaemic stroke occurrence with COVID-19 infection in the context of a systemic highly prothrombotic state [4].

A study of hospitalized patients in New York suggested patients with COVID-19 and stroke had significantly higher mortality than controls, but the study included only 32 patients with ischaemic stroke and COVID-19 [5]. An analysis of 174 patients hospitalized with acute ischaemic stroke and COVID-19, 1:1 propensity score matched to historical controls with ischaemic stroke from a single site, suggested that COVID-19-associated ischaemic strokes were more severe, with worse functional outcomes and higher odds of mortality (odds ratio for mortality 4.3 [95\% confidence interval 2.22-8.30]) [6]. Further evidence is needed to confirm this finding. Overall, there are limited available data on the outcomes of ischaemic stroke patients with COVID-19 compared to outcomes of ischaemic stroke patients without COVID-19. In the current study, the aim was to determine the association between COVID-19 and mortality for patients with ischaemic stroke in a large multicentre study.

\section{Materials and Methods}

This study utilized TriNetX, a global federated health research network providing access to statistics on electronic medical records (EMRs). To gain access to the data, a request can be made to TriNetX (https://live.trinetx.com), but costs may be incurred, and a data-sharing agreement is needed. The data within TriNetX is provided by healthcare organizations (HCOs) including academic medical centres, specialty physician practices, and community hospitals. Available data include demographics, diagnoses (using International Classification of Diseases, Tenth Revision, Clinical Modification [ICD-
10-CM] codes), procedures, medications, and measurements. To comply with legal frameworks and ethical guidelines guarding against data re-identification, the identity of participating HCOs and their individual contribution to each dataset are not disclosed. The TriNetX platform only uses aggregated counts and statistical summaries of de-identified information. No protected health information or personal data is made available to the users of the platform.

The TriNetX network was searched for patients meeting the inclusion criteria of this study on December 2, 2020. At the time of analysis, there were 56 HCOs in the TriNetX network used in this study ( $90 \%$ of HCOs from the USA and $10 \%$ from outside of the USA). From these, $48 \mathrm{HCOs}$ had patients which met the inclusion criteria and were included in the present study. The inclusion criteria were patients in the network with the following recorded in their EMRs: (1) COVID-19 between January 20, 2020, and October 1, 2020, (2) ischaemic stroke (ICD-10-CM code I63) within 30 days of COVID-19 recorded in EMRs by positive test or ICD-10-CM code, and (3) inpatient within 30 days of COVID-19 recorded in EMRs using the following "visit" terms in their EMRs: "Initial Hospital Inpatient Care Services," "Critical Care Services," "Inpatient Encounter," "Inpatient Acute," "Inpatient Short Stay," or "Inpatient Non-Acute." The search allowed for 60 days follow-up for all participants. January 20,2020 , was chosen as the start date because the first confirmed case of COVID-19 reported in the USA was reported in Washington State on January 20, 2020, and the cohort was predominately from HCOs within the USA [7]. The inclusion criteria for historical controls were patients in the network with the following recorded in their EMRs: (1) ischaemic stroke (ICD-10-CM code I63) between January 20, 2019, and October 1, 2019, and (2) inpatient 2 weeks before or 2 weeks after ischaemic stroke using the following "visit" terms in their EMRs: "Initial Hospital Inpatient Care Services", "Critical Care Services," "Inpatient Encounter," "Inpatient Acute," "Inpatient Short Stay," or "Inpatient Non-Acute."

COVID-19 status was determined using codes in EMRs or a positive test result identified with COVID-19 specific laboratory codes. Patients with COVID-19 were identified following criteria provided by TriNetX based on Centres for Disease Control and Prevention (CDC) coding guidelines [8]. Patients were included if they had 1 or more of the following ICD-10-CM codes in their EMRs: U07.1 COVID-19; U07.2 COVID-19, virus not identified; B97.29 other coronavirus as the cause of diseases classified elsewhere; B34.2 coronavirus infection, unspecified; or a positive test result identified with COVID-19 specific laboratory Logical Observation Identifiers Names and Codes (LOINCs). Patients with ICD Ninth Revision (ICD-9) code 079.89 were excluded to reduce the likelihood of patients with false-positive COVID-19 because this code may still be used occasionally as a "catch-all" code for $>50$ viral infections.

No sample size calculations were performed. Baseline characteristics were compared using a $\chi^{2}$ test for categorical variables and an independent samples $t$ test for continuous variables within the TriNetX platform. The TriNetX platform was used to run 1:1 propensity score matching using logistic regression. The platform uses nearest-neighbour matching with a tolerance level of 0.01 and difference between propensity scores $\leq 0.1$. Variables included in propensity score matching were age, sex, race, and history of hypertension, cerebrovascular disease, ischaemic heart disease, chronic obstructive pulmonary disease, diabetes mellitus, atrial fibrillation and flutter, liver disease, chronic kidney disease (CKD), neoplasms, and mental disorders associated with physiological conditions. These variables were chosen because they are established risk 
Table 1. Baseline characteristics for patients with ischaemic stroke and COVID-19 and historical controls with ischaemic stroke without COVID-19 before and after propensity score matchings

\begin{tabular}{|c|c|c|c|c|c|c|}
\hline \multirow[t]{2}{*}{$\%(N)$} & \multicolumn{3}{|l|}{ Initial population } & \multicolumn{3}{|c|}{ Propensity score-matched populations } \\
\hline & $\begin{array}{l}\text { stroke and no } \\
\text { COVID-19 } \\
(n=48,363)\end{array}$ & $\begin{array}{l}\text { stroke and } \\
\text { COVID-19 } \\
(n=954)\end{array}$ & $p$ value & $\begin{array}{l}\text { stroke and no } \\
\text { COVID-19 } \\
(n=952)\end{array}$ & $\begin{array}{l}\text { stroke and } \\
\text { COVID-19 } \\
(n=952)\end{array}$ & $p$ value \\
\hline Age, years, mean (SD) & $68.6(15.6)$ & $67.3(15.4)$ & 0.008 & $67.7(14.9)$ & $67.3(15.4)$ & 0.49 \\
\hline Female $^{a}$ & $49.0(23,708)$ & $43.0(410)$ & 0.0002 & $42.0(400)$ & $43.1(410)$ & 0.64 \\
\hline \multicolumn{7}{|l|}{ Race $^{\mathrm{b}}$} \\
\hline White & $69.7(33,717)$ & $48.8(466)$ & $<0.0001$ & $48.7(464)$ & $48.8(466)$ & 0.93 \\
\hline Black or African American & $17.1(8,305)$ & $30.2(288)$ & $<0.0001$ & $31.3(298)$ & $30.2(288)$ & 0.62 \\
\hline Asian & $1.4(675)$ & $2.1(20)$ & 0.70 & $1.7(16)$ & $2.1(20)$ & 0.50 \\
\hline Native Hawaiian or other Pacific Islander & $0.1(41)$ & $1.1(10)$ & $<0.0001$ & $0(0)$ & $1.1(10)$ & 0.002 \\
\hline American Indian or Alaska Native & $0.5(233)$ & $1.1(10)$ & 0.01 & $1.1(10)$ & $1.1(10)$ & 1.00 \\
\hline Unknown & $11.1(5,392)$ & $17.7(169)$ & $<0.0001$ & $17.2(164)$ & $17.7(169)$ & 0.86 \\
\hline Hypertension & $58.2(28,140)$ & $67.0(639)$ & $<0.0001$ & $68.6(653)$ & $67.0(638)$ & 0.46 \\
\hline Atrial fibrillation and flutter & $18.3(8,827)$ & $24.1(230)$ & $<0.0001$ & $22.7(216)$ & $24.2(230)$ & 0.45 \\
\hline Cerebrovascular disease & $44.0(21,299)$ & $52.1(497)$ & $<0.0001$ & $52.5(500)$ & $52.2(497)$ & 0.89 \\
\hline Ischaemic heart disease & $29.4(14,206)$ & $36.7(350)$ & $<0.0001$ & $36.5(347)$ & $36.7(349)$ & 0.92 \\
\hline \multicolumn{7}{|l|}{ Mental disorders associated with } \\
\hline physiological conditions & $10.4(5,029)$ & $17.6(168)$ & $<0.0001$ & $17.3(165)$ & $17.6(168)$ & 0.86 \\
\hline Chronic obstructive pulmonary disease & $12.8(6,197)$ & $16.2(155)$ & 0.002 & $18.2(173)$ & $16.3(155)$ & 0.27 \\
\hline Liver disease & $8.6(4,154)$ & $13.4(128)$ & $<0.0001$ & $12.1(115)$ & $13.4(128)$ & 0.37 \\
\hline Diabetes mellitus & $29.4(14,221)$ & $42.2(403)$ & $<0.0001$ & $44.0(419)$ & $42.2(403)$ & 0.46 \\
\hline CKD & $18.8(9,077)$ & $27.6(263)$ & $<0.0001$ & $27.5(262)$ & $27.6(263)$ & 0.96 \\
\hline Neoplasms & $25.6(12,387)$ & $30.6(292)$ & 0.0001 & $27.6(263)$ & $30.7(292)$ & 0.14 \\
\hline
\end{tabular}

Baseline characteristics were compared using a $\chi^{2}$ test for categorical variables and an independent samples $t$ test for continuous variables. COVID-19: coronavirus disease 2019; HCO, healthcare organization. ${ }^{a}$ Sex unknown for $0.1 \%$ of patients. ${ }^{b}$ Data are taken from structured fields in the electronic medical record systems of the participating HCOs; therefore, there may be regional or country-specific differences in how race categories are defined.

factors for mortality and were significantly different between the cohorts with and without COVID-19. Kaplan-Meier survival curves for all-cause 60-day mortality by COVID-19 status were produced. Statistical significance was set at $p<0.05$. All analyses were conducted within the TriNetX online platform.

\section{Results}

On the date of the search, there were 954 patients with ischaemic stroke and COVID-19 and 48,363 patients with ischaemic stroke without COVID-19 in the same time period in 2019 (historical controls) identified with the inclusion criteria. Of the patients with COVID-19, 91.2\% $(n=$ 870 ) were identified from the ICD-10-CM code U07.1 or positive test results identified with COVID-19-specific laboratory LOINCs. Compared to patients with ischaemic stroke without COVID-19, patients with COVID-19 had a lower prevalence of white patients (48.8 vs. $69.7 \%, p<$ 0.0001 ) and a higher prevalence of black or African Amer- ican patients ( 30.2 vs. $17.1 \%, p<0.0001)$. The mean age of the patients without COVID-19 was statistically significantly higher than that of patients with COVID-19 (mean [SD] 68.6 [15.6] vs. 67.3 [15.4], $p=0.008$ ). Patients with ischaemic stroke and COVID-19 had a statistically higher prevalence of history of hypertension, cerebrovascular disease, diabetes mellitus, ischaemic heart disease, atrial fibrillation, CKD, chronic obstructive pulmonary disease, liver disease, neoplasms, and mental disorders due to known physiological conditions (Table 1).

After propensity score matching, there were 952 cases and 952 historical controls, and the cases and historical controls were better balanced on all included characteristics (all $p>0.05$, apart from the proportions of people recorded as Native Hawaiian or other Pacific Islander remained higher for patients with ischaemic stroke and COVID-19 (1.1 vs. $0 \%, p=0.002)$ ). After propensity score matching, KaplanMeier survival analysis showed the survival probability was significantly lower in ischaemic stroke patients with COVID-19 (78.3 vs. 91.0\%, log-rank test $p<0.0001$, Fig. 1 ). The 


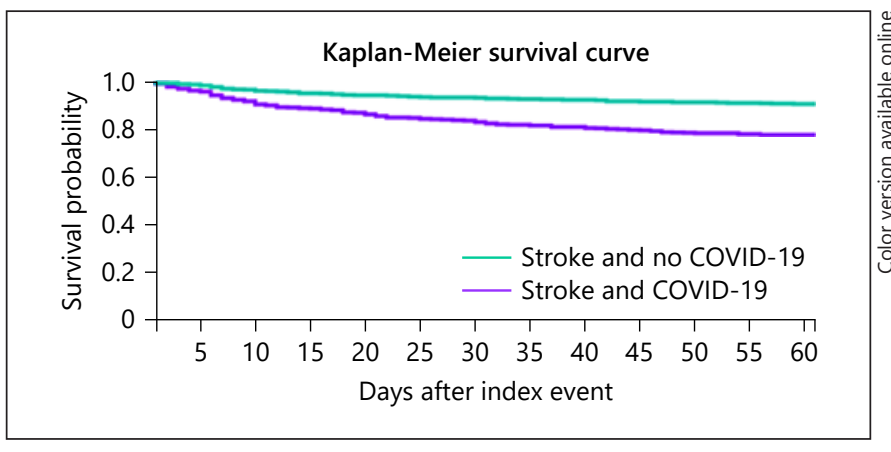

Fig. 1. Kaplan-Meier survival curve of all-cause 60-day mortality for ischaemic stroke patients with and without COVID-19 after propensity score matching. Propensity score matching for age, sex, race, and history of hypertension, cerebrovascular disease, ischaemic heart disease, chronic obstructive pulmonary disease, diabetes mellitus, atrial fibrillation and flutter, liver disease, CKD, neoplasms, and mental disorders associated with physiological conditions. Purple line is patients with ischaemic stroke without COVID-19, and green line is patients with ischaemic stroke without COVID-19. COVID-19, coronavirus disease 2019.

odds of 60-day mortality were significantly higher for patients with ischaemic stroke and COVID-19 compared to the propensity score-matched historical controls (odds ratio: 2.51 [95\% confidence interval 1.88-3.34]).

\section{Discussion}

In the present study of over 1,900 patients with ischaemic stroke from multiple HCOs, patients with ischaemic stroke with COVID-19 had significantly higher 60day all-cause mortality than historical controls with ischaemic stroke without COVID-19, propensity score matched for age, sex, race, and many comorbidities. While the relationship of venous thromboembolism and a prothrombotic state is well-recognized, increasing focus has been directed towards large vessel arterial thrombosis, including myocardial infarction among patients with COVID-19 [3]. Indeed, cerebrovascular disease has been associated with higher risk of transfer to the intensive care unit among COVID-19 patients, and large-vessel stroke has been reported in younger COVID-19 patients $[9,10]$. Ischaemic stroke in such patients is increasingly recognized $[4,9]$, but it has been unclear whether COVID-19 per se increased the risk of mortality for ischaemic stroke patients. The results of this study suggest patients with ischaemic stroke and COVID-19 may have a higher risk of 60-day mortality compared to ischaemic stroke patients without COVID-19. This is consistent with evi- dence from a small study of patients with ischaemic stroke in New York, showing those with COVID-19 had significantly higher mortality than controls [5]. Similar to the present analysis, a previous study has been conducted of 174 patients hospitalized with acute ischaemic stroke and COVID-19 from multiple sites 1:1 propensity score matched to historical controls from a single site. The results of the previous study showed COVID-19-associated ischaemic strokes were more severe with worse functional outcomes and higher odds of mortality compared to historical controls with ischaemic strokes without COVID-19 (odds ratio for mortality 4.3 [95\% confidence interval 2.22-8.30]) [6]. By utilizing a larger sample size and historical controls from multiple HCOs, the results from the present study add to the evidence base that ischaemic strokes are associated with higher mortality than nonCOVID-19 ischaemic strokes. The odds ratio for mortality in the previous study was higher than in the present study but the confidence intervals were wider and include the odds ratio for mortality identified in the present study (odds ratio: 2.51 [95\% confidence interval 1.88-3.34]).

Furthermore, a recent pooled analysis of 4 studies suggested a 2.5-fold increase in the odds of severe COVID-19 illness for patients with a history of cerebrovascular disease [11]. Yet, in the same review, while a trend was found, there was no statistically significant association of stroke with mortality in patients with COVID-19 infection; however, only 2 studies were included in this analysis [11]. These 2 included studies examined whether stroke was a risk factor for mortality among patients with COVID-19 [12,13] rather than comparing the risk of mortality among patients with stroke with and without COVID-19. In a subsequent metaanalysis of 8 studies, cerebrovascular disease was identified to be significantly associated with an increased risk of mortality in patients with COVID-19 [14]. Additionally, a recent study of over 20,000 hospitalized patients with COVID-19 in the UK reported that presence of a chronic neurological disorder (such as stroke) was associated with increased in-hospital mortality [15]. Conversely, history of cerebrovascular disease was not associated with higher mortality among over 31,000 patients with COVID-19 from 24 HCOs in the USA [16]. In the present study, there was a higher proportion of non-white patients among the COVID-19 strokes compared to non-COVID-19 strokes, consistent with racial/ethnic disparities in outcomes among non-white patients relating to COVID-19 [17].

The present study has limitations including that the data were collected from the HCO EMRs and some comorbidities may be underreported. Previous studies have shown recording of ICD codes in EMRs may vary by fac- 
tors such as age, number of comorbidities, severity of illness, length of hospitalization, and whether in-hospital death occurred [18]. Race was not available for all participants and was based on limited prespecified race categories within TriNetX. Important information about the stroke severity of the participants could not be determined from the available data. Stroke severity scales such as the National Institutes of Health Stroke Scale (NIHSS) and assessments for disability following stroke, such as the modified Rankin Scale (mRS), should be included where possible for future studies examining the impact of COVID-19 for patients with ischaemic stroke. Further residual confounding may include factors such as BMI, which was not available for the majority of participants. It could also not be determined if there was any impact of attending different HCOs due to data privacy restrictions. All deaths of the included patients captured within the TriNetX network were examined; however, deaths outside of the participating HCOs are not well-captured. The study attempted to include only patients who had a stroke following COVID-19 by only including patients with ischaemic stroke recorded in their EMRs up to 30 days following COVID-19, but it is uncertain if participants had COVID-19 prior to their stroke or whether it was acquired following their stroke. This could have important impacts on outcomes for the patients and should be explored further in prospective studies. It is unclear if the results are generalizable to the wider population of the USA, as at the time of analysis, there were 56 HCOs in the TriNetX network used in this study (90\% of the HCOs from the USA and 10\% from outside of the USA).

\section{Conclusion}

This study utilized a large cohort of patients with ischaemic stroke and COVID-19 and propensity score matched them to historical controls with ischaemic stroke without COVID-19 from multiple sites. The results suggest that COVID-19 is associated with higher 60-day mortality for patients with ischaemic stroke. The study was based on EMRs and has limitations but adds to the evidence base that COVID-19 worsens outcomes for patients with ischaemic stroke. Further research should examine interventions which reduce risk of mortality for patients with ischaemic stroke and COVID-19.

\section{Statement of Ethics}

As a federated network, TriNetX did not require ethical approvals as no patient identifiable identification is received.

\section{Conflict of Interest Statement}

G.Y.H.L.: consultant for Bayer/Janssen, Bristol-Myers Squibb (BMS)/Pfizer, Medtronic, Boehringer Ingelheim, Novartis, Verseon, and Daiichi-Sankyo and speaker for Bayer, BMS/Pfizer, Medtronic, Boehringer Ingelheim, and Daiichi-Sankyo. No fee was directly received. D.A.L. has received an investigator-initiated educational grant from BMS, has been a speaker for Boehringer Ingelheim and BMS/Pfizer, and has consulted for BMS, Boehringer Ingelheim, and Daiichi-Sankyo. E.F.E. and P.U.: employed by TriNetX Inc. S.L.H.: none declared.

\section{Funding Sources}

No specific funding was received for this study. TriNetX Inc. funded the acquisition of the data used.

\section{Author Contributions}

S.L.H. and G.Y.H.L. made a substantial contribution to the design of the work. E.F.E. and P.U. made substantial contributions to the acquisition of the data. S.L.H. analyzed the data. S.L.H., E.F.E., D.L., and G.Y.H.L. made a substantial contribution to the interpretation of the data. S.L.H. drafted the work, and all other co-authors revised it critically for important intellectual content.

\section{References}

1 World Health Organization. Novel coronavirus (2019-nCoV) situation Report: 11. 2020.

2 European Centre for Disease Prevention and Control. Situation update worldwide, as of 7 July 2020. 2020.

3 Bikdeli B, Madhavan MV, Jimenez D, Chuich T, Dreyfus I, Driggin E, et al. COVID-19 and thrombotic or thromboembolic disease: implications for prevention, antithrombotic therapy, and follow-up. J Am Coll Cardiol. 2020 Jun 16;75(23):2950-73.
4 Beyrouti R, Adams ME, Benjamin L, Cohen $\mathrm{H}$, Farmer SF, Goh YY, et al. Characteristics of ischaemic stroke associated with COVID-19. J Neurol Neurosurg Psychiatry. 2020 Aug;91(8):889-91.

5 Yaghi S, Ishida K, Torres J, Mac Grory B, Raz E, Humbert K, et al. SARS2-CoV-2 and stroke in a New York healthcare system. Stroke. 2020 Jul;51(7):2002-11.

6 Ntaios G, Michel P, Georgiopoulos G, Guo Y, Li W, Xiong J, et al. Characteristics and outcomes in patients with COVID-19 and acute ischemic stroke: the global COVID-19 stroke registry. Stroke. 2020 Sep 1;51(9): e254-e58.

7 Holshue ML, DeBolt C, Lindquist S, Lofy $\mathrm{KH}$, Wiesman J, Bruce H, et al. First case of 2019 novel coronavirus in the United States. N Engl J Med. 2020 Mar 5;382(10): 929-36.

8 Centers for Disease Control and Prevention. ICD-10-CM official coding guidelines: supplement coding encounters related to COVID-19 coronavirus outbreak. 2020. 
9 Oxley TJ, Mocco J, Majidi S, Kellner CP, Shoirah H, Singh IP, et al. Large-vessel stroke as a presenting feature of Covid-19 in the young. N Engl J Med. 2020 May 14;382(20):e60.

10 Wang D, Hu B, Hu C, Zhu F, Liu X, Zhang J, et al. Clinical characteristics of 138 hospitalized patients with 2019 novel coronavirus-infected pneumonia in Wuhan, China. JAMA. 2020;323(11):1061-9.

11 Aggarwal G, Lippi G, Michael Henry B. Cerebrovascular disease is associated with an increased disease severity in patients with coronavirus disease 2019 (COVID-19): a pooled analysis of published literature. Int J Stroke. 2020 Jun 1;15(4):385-9.
12 Ruan Q, Yang K, Wang W, Jiang L, Song J. Clinical predictors of mortality due to COVID-19 based on an analysis of data of $150 \mathrm{pa}$ tients from Wuhan, China. Intensive Care Med. 2020;46(5):846-8.

13 Yang X, Yu Y, Xu J, Shu H, Xia J, Liu H, et al. Clinical course and outcomes of critically ill patients with SARS-CoV-2 pneumonia in Wuhan, China: a single-centered, retrospective, observational study. Lancet Respir Med. 2020 May;8(5):475-81.

14 Wang Y, Shi L, Wang Y, Duan G, Yang H. Cerebrovascular disease is associated with the risk of mortality in coronavirus disease 2019. Neurol Sci. 2020 Aug;41(8):2017-19.
15 Docherty AB, Harrison EM, Green CA, Hardwick HE, Pius R, Norman L, et al. Features of 20,133 UK patients in hospital with covid-19 using the ISARIC WHO clinical characterisation protocol: prospective observational $\mathrm{CO}^{-}$ hort study. BMJ. 2020;369:m1985.

16 Harrison SL, Fazio-Eynullayeva E, Lane DA, Underhill P, Lip GYH. Comorbidities associated with mortality in 31,461 adults with COVID-19 in the United States: a federated electronic medical record analysis. PLoS Med. 2020;17(9):e1003321.

17 Webb Hooper M, Napoles AM, Perez-Stable EJ. COVID-19 and racial/ethnic disparities. JAMA. 2020 Jun 23;323(24):2466-7.

18 Chong WF, Ding YY, Heng BH. A comparison of comorbidities obtained from hospital administrative data and medical charts in older patients with pneumonia. BMC Health Serv Res. 2011;11:105-5. 